Bayan A Hassan BDS, MSc PhD (Lec)

\section{Effect of various surface treatment procedures on shear bond strength of stainless-steel orthodontic brackets to composite resin}

Department of Pediatric Orthodontic Preventive dentistry College of Dentistry, Hawler Medical University

\begin{abstract}
الخلاصة
الاهداف: تهذف الدراسة تقييم قوة قص الحاصر ات التقويمية المثبتة على الحشوات الر اتينجية المركبة باستخدام اربعة انواع من العلاجات

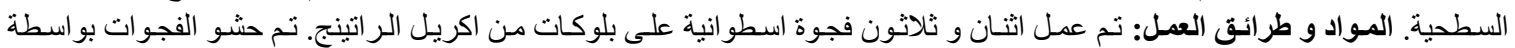

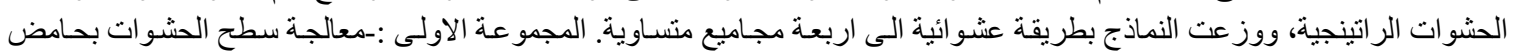

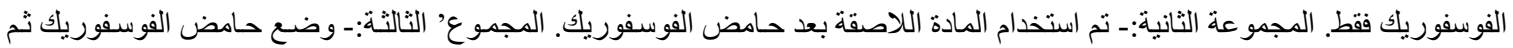

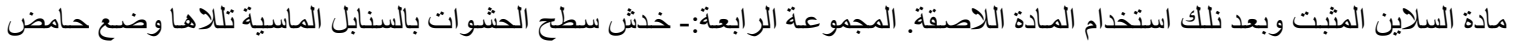

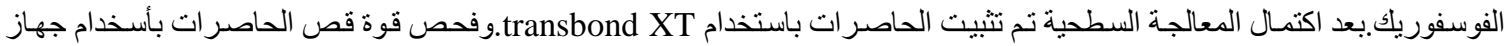

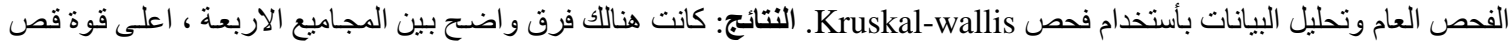

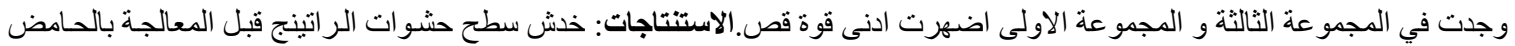

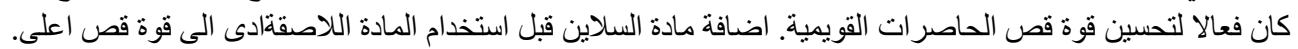

\begin{abstract}
Aims: The aim of this study was to evaluate the shear bond strength of brackets bonded to composite restorations using four types of surface conditioning procedure. Materials and Methods: Thirty two cylindrical cavities were made at the center of self-cured acrylic resin blocks. All cavities were then filled with composite resin. The specimens were randomly divided into four groups. Group one: Composite surface was acid etched with 37\% phosphoric acid. Group two: After using 37\% phosphoric acid, bonding agent was applied and cured. Group three: After using 37\% phosphoric acid silane coupling agent was applied then bonding agent was applied. Group Four: The composite surface roughened with a diamond bur, and then $37 \%$ phosphoric acid was applied. Brackets were bonded onto the composite prepared samples with Transbond XT. Shear Bond Strength was measured by a universal testing machine. KruskalWallis test was utilized for data analysis. Results: There was a significant difference between the four groups. The highest bond strength was that of Group 3. Group 1 had the lowest bond strength. Conclusions: Roughening the composite surface before acid etching was effective to improve the bond strength of orthodontic brackets to composite restorations. Addition of silane before bonding agent application result in highest bond strength.
\end{abstract}

Key words: Orthodontic bracket, surface treatment, shear bond strength, composite restoration.

Hassan BA. Effect of various surface treatment procedures on shear bond strength of stainless-steel orthodontic brackets to composite resin. Al-Rafidain Dent J. 2018, 18(1):21-30.

Received: 23/4/2018 Sent to Referees: 6/5/2018 Accepted for Publication: 3/6/2018

\section{INTRODUCTION}

Nowadays, the seeking for orthodontic treatment increases and the adults compose a significant number of orthodontic patients. Therefore, bonding the metal orthodontic brackets to composite resin restorations became a common procedure in the daily orthodontic practice 
(1-6). When the old composite resin restoration has been polished or contaminated, the bond strength to new composite resin restoration is significantly reduced. ${ }^{(4,6-7)}$.Number of techniques have been suggested in order to maximize the bond strength between the two composite restorations, such as acid etching, micro etching and the use of chemical agents ${ }^{(8-10)}$.

Many studies, in restorative dentistry have been focused on repairing composite resin restoration by using different techniques. Unlike the restorative dentistry, in orthodontic treatment maximum adhesion to the surface of the composite resin restoration is very important, it has been suggested that bond strength of 6-10 $\mathrm{MPa}$ are sufficient. ${ }^{(11)}$

Although brackets bonded to the freshly roughened surface of an old composite restoration have had clinical success, some authors recommend an intermediate primer as well ${ }^{(12)}$. Excite (Ivoclar.Vivadent, Schaan, Liechtenstein) is a fifth-generation, light-activated dentin bonding agent that sometimes called one bottle system. It is recommended for direct bonding of resin composite. This type of adhesive combines the primer and bonding agent into a single solution and is acetonefree. A separate etching step still is required (13). According to the manufacturer instructions, Excite is distinguished from other "one-component" bonding agents in that it contains extremely small (i.e., 12nanometer) filler particles. Because they are so small, the manufacturer claims that the filler particles can penetrate into the demineralized dentin and contribute to the formation of the hybrid layer. Another reported advantage of their small size is that they do not contribute significantly to the adhesive's film thickness. ${ }^{(14)}$

Accordingly, a limited number of studies have been reported in the literature, the saline agent in such situations has been an encouraging subject, This investigation was undertaken to assess the shear bond strength SBS of stainless steel brackets when using a saline coupling agent applied to resin composite surfaces.

\section{MATERIALS AND METHODS}

A total thirty two cylindrical cavities 8 $\mathrm{mm}$ in diameter and $2 \mathrm{~mm}$ in depth were made at the center of self-cured acrylic resin blocks which were poured in metal mold 2 $\mathrm{cm}$ in diameter and $2.5 \mathrm{~cm}$ in depth. All cavities were then filled with anterior composite resin (Shofu, japan) shade A2. The composite resin was packed against the cavity with Ash plastic instrument to avoid air entrapment and then treated with light cure for 20 second by light curing unit (Astralis 5, Leichenstein, Germany). The specimens were randomly divided into four groups of 8 samples; 
Group one: The composite surface was acid etched with $37 \%$ phosphoric acid for 30 seconds then the composite surface washed for 15 seconds with copious water to remove the acid and air dried with oil free air for 10 seconds. After that, the maxillary central stainless steel brackets (Dentarum, Germany) were bonded by applying a constant force with the help of a surveyor. Transbond XT (3M, ESPE, Minnesota, USA) was used according to the manufacturer instructions. Excess composite was gently removed before curing and the composite light cured from all surfaces for 10 seconds for each side (mesially, distally, occusaly and gingivaly) with light curing unit.

Group two: after using 37\% phosphoric acid and rinsing with copious water as described in group one, Excite bonding agent (Ivoclar, Vivadent, Schaan, Leichtenstein) was applied by a micro brush on the surface of the composites resin that were treated. After light curing of the Excite bonding agent for 20 seconds, the brackets were bonded as the same manner in group one.

Group three: after using 37\% phosphoric acid and rinsing with copious water as described in group one, silane coupling agent was applied with brush to the composite surface and left for one minute then Excite bonding agent (Ivoclar,
Vivadent, Schaan, Leichtenstein) was applied in the same manner as described in group two.

Group Four: the composite surface was roughened with a diamond bur (Shofu, Japan), then $37 \%$ phosphoric acid was applied to the surface of the composite resin for 30 seconds and the brackets were bonded. All procedures were performed as described previously for the group one. Then all the specimens stored in an incubator for 72 hours at $37^{\circ} \mathrm{C}$ and $100 \%$ humidity.

\section{Shear bond strength testing:}

Following storage, each sample was fitted into a specially constructed jag and subjected to bond testing with a universal testing machine. Using knife edge rod of 0.5 $\mathrm{mm}$ width with a cross head speed 0.5 $\mathrm{mm} / \mathrm{min}$ that applied vertically across composite and bracket interface. The force that was required to shear the brackets was recorded in Newton measurements. The shear bond strengths were calculated in mega pascals (MPa) by dividing the force to the surface area of the base of the brackets (12mm).

\section{Statistical Analysis:}

The data were collected and analyzed statistically, the data tested for normal distribution and since the data were not 


\section{Hassan BA}

normally distributed then it was decided to apply Kruskal-wallis test. The level of significance for all tests was determined at $\mathrm{P}<0.05$.

\section{RESULTS}

The descriptive statistic data of the shear bond strength test of the different groups was shown in Table 1.

Table (1): Descriptive statistic of shear bond strength for all groups.

\begin{tabular}{cccccc}
\hline & & & & & Mean \\
\cline { 2 - 4 } & $\mathrm{N}$ & Minimum & Maximum & $\begin{array}{c}\text { Std. } \\
\text { Deviation }\end{array}$ \\
\hline Group1 & 8 & 7.50 & 10.83 & 9.5820 & 1.40735 \\
Group2 & 8 & 14.17 & 19.17 & 16.7696 & 2.06281 \\
group3 & 8 & 20.00 & 23.33 & 21.8740 & 1.45951 \\
group4 & 8 & 15.00 & 19.17 & 16.8748 & 1.87641 \\
Valid N & 8 & & & & \\
(listwise) & & & & & \\
\hline
\end{tabular}

The highest bond strength was that of Group 3, which used silane and bonding in addition to acid. Group 1 had the lowest bond strength, which used only acids etch technique before bonding the bracket.
According to the Kruskal-Wallis test there was a significant difference in shear bond strength among all different groups Table (2).

Table (2): Independent samples Kruskal - Wallis test

Hypothesis Test Summary

\begin{tabular}{|lllll|}
\hline & Null Hypothesis & Test & Sig. & Decision \\
\hline 1 & $\begin{array}{l}\text { The distribution of reading is the } \\
\text { same across categories of Group. }\end{array}$ & $\begin{array}{l}\text { Independent- } \\
\text { Samples } \\
\text { Kruskal- } \\
\text { Wallis Test }\end{array}$ & .0oo & $\begin{array}{l}\text { Reject the } \\
\text { null } \\
\text { hypothesis. }\end{array}$ \\
\hline
\end{tabular}

Asymptotic significances are displayed. The significance level is .05 .

Further analysis using Bonferroni correction for multiple test revealed that shear bond strength value among one and three were statistically highly significant at $\mathrm{p}<0.01$ as shown in Table 3. 
Table (3): Bonferroni correction for multiple test

Pairwise Comparisons of Group

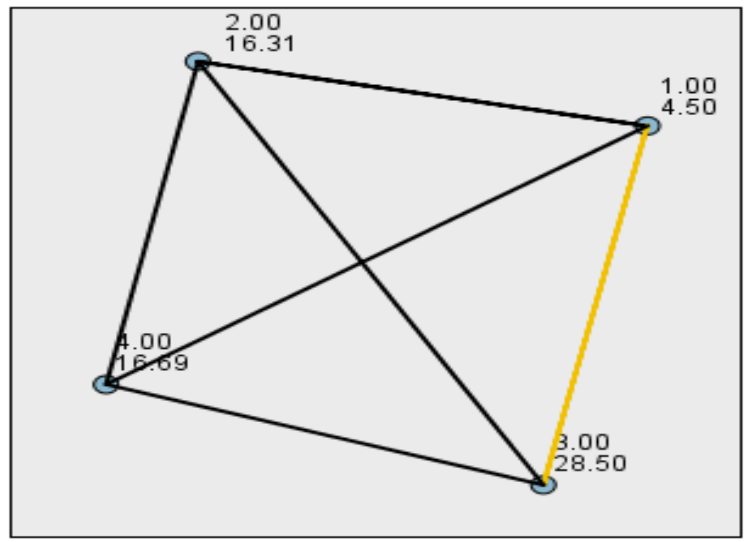

Each node shows the sample average rank of Group.

\begin{tabular}{|lrrrrr|}
\hline Sample1-Sample2 & $\begin{array}{c}\text { Test } \\
\text { Statistic }\end{array}$ & $\begin{array}{c}\text { Std. } \\
\text { Error }\end{array}$ & $\begin{array}{c}\text { Std. Test } \\
\text { Statistic }\end{array}$ & \multicolumn{1}{c|}{ Sig. } & \multicolumn{1}{c|}{ Adj.Sig. } \\
\cline { 2 - 6 } $\mathbf{1 . 0 0 - 2 . 0 0}$ & -11.812 & 4.678 & -2.525 & .012 & .069 \\
\hline $\mathbf{1 . 0 0 - 4 . 0 0}$ & -12.188 & 4.678 & -2.605 & .009 & .055 \\
\hline $\mathbf{1 . 0 0 - 3 . 0 0}$ & -24.000 & 4.678 & -5.130 & .000 & .000 \\
\hline $\mathbf{2 . 0 0 - 4 . 0 0}$ & -.375 & 4.678 & -.080 & .936 & 1.000 \\
\hline $\mathbf{2 . 0 0 - 3 . 0 0}$ & -12.188 & 4.678 & -2.605 & .009 & .055 \\
\hline $\mathbf{4 . 0 0 - 3 . 0 0}$ & 11.812 & 4.678 & 2.525 & .012 & .069 \\
\hline
\end{tabular}

Each row tests the null hypothesis that the Sample 1 and Sample 2 distributions are the same.

Asymptotic significances (2-sided tests) are displayed. The significance level

is .05.

Significance values have been adjusted by the Bonferroni correction for multiple tests.

\section{DISCUSSION}

The increased need of adults to orthodontic treatment results in the necessity for orthodontists to fix brackets and tubes on composite restorations. When bonding orthodontic brackets to composite surfaces, it is necessary to alter the inert characteristics of the surfaces to achieve clinically acceptable bond strength. Bond strength should be high enough to resist accidental debonding during treatment, but also low enough to remove the bracket from the composite surfaces without generating excessive force which might damage the restoration ${ }^{9}$.

Laboratory methods have been proposed to simulate oral conditions and facilitate comparisons among different dental materials as clinical studies that assess the shear bond strength of orthodontic brackets are extremely difficult to be conducted. It is virtually impossible to standardize the oral status of each patient, 


\section{Hassan BA}

which prevents the prediction of durability and the comparison of dental materials. ${ }^{(14)}$

In the present study, four procedures have been used for preparing the composite surface before the bonding of brackets in order to improve the bond strength of new composite restorations to previous composite fillings.

The results of the current studies showed highly significant differences between group1 which used acid etch only before adhesive resin application and group 3 which treated with silane coupling agent in addition to Exite bonding agent application.

Further studies found that the conventional acid etching with $37 \%$ phosphoric acid created lower bond strength values. ${ }^{(15-}$ 17), which is in agreement with the present study.

It was demonstrated that orthophosphoric acid with a concentration of $37 \%$ is not able to etch a ceramic surface and, consequently, does not produce physical or topographical changes on porcelain ${ }^{(18) .}$

In the present study, also bond strength was achieved with the application of Excite bonding agent and acid etching on the composite surface. As it has role in improving surface wetting of composite restoration surfaces that act as a bonding agent which can lead to the formation of more resin tags. Increasing the number of resin tags improves the shear bond strength of brackets to composite restorations. It seems that roughening the composite surface provides conditions for Excite bonding agents to form more resin tags and increase bond strength.$^{(14)}$

In the present study, it is found that surface roughening with a diamond bur plus application of $37 \%$ phosphoric acid achieved bond strengths that should be clinically successful. This finding is similar to study by Eslamian et $\mathrm{al}^{(19)}$ who found that, when stainless steel brackets are bonded to composite restorations, treated with diamond bur. There is significant higher shear bond strength than those, bonded to composite surfaces treated with hydrofluoric acid, roughening with a diamond bur improved retentive surfaces.

The findings of Chay et $\mathrm{al}^{20}$ support the claim that treatment, such as roughening with greenstone or sandblasting the surface of the provisional material, produced increased bond strength of orthodontic brackets after artificial aging. Another study concluded that the preparation of the porcelain surface with sandblasting is better than that with a diamond bur. ${ }^{(21}$ ) Because of the risk of damaging the porcelain during pretreatment or debonding, roughening of the surfaces must be avoided ${ }^{(22-24)}$.

One of the studies that disagrees with this finding and suggests that surface roughening without silane treatment may not 
produce clinically acceptable bond strengths ${ }^{(25)}$.

Another founding achieved in this study was the influence of silane on the shear bond strength between orthodontic brackets and bonding materials. Highest mean value (21.87) was found in groups that treated with silane and Exite application. Silane coupling agents are usually monomeric species in which silicon is linked to reactive organic radicals and hydrolyzable ester groups. The reactive organic groups become chemically bonded to the resin molecules. Hydrolyzable monovalent groups bond chemically to silicon contained in the glass matrix and lithium disilicate crystals ${ }^{(26,27)}$. Then, a chemical bond is formed between the silane coupling agent and silica layer on the ceramic surface or the bonding materials. Hisamatsu et $\mathrm{al}^{28}$ concluded that no matter what variation in the material, the mixing of a silane primer with bonding agent usually demonstrated the greatest degree of bond strength. Barbosa et $\mathrm{al}^{29}$ found that surface roughening with a diamond bur followed by the silane application produces clinically acceptable bond strengths. In contradiction, other studies revealed that the use of silane did not significantly affect the bond strength and may be an unnecessary additional step $(23,30,31)$. The result of this study was in disagreement with the previous study ${ }^{32}$ who reported that using a silane agent offered no advantage when bonding orthodontic brackets to composite restorations.

The results of this in vitro study indicated that the use of silane increases the bond strength values; and conditioning with $37 \%$ phosphoric acid for $30 \mathrm{~min}$, followed by the application of silane and bonding agent was considered the best composite preparation method. These results are in agreement with other studies ${ }^{(33-36)}$.The use of silane prior to bonding was the single most important factor in determining satisfactory bond strength ${ }^{(37) \text {. }}$

\section{CONCLUSIONS}

It was concluded that the bond that contain silane coupling agent will raise the bond strength between composite increment, also surface roughness of composite surface raise bond strength between composite increment better than composite smooth surface.

\section{REFERENCES}

1. Gottlieb EL, Nelson AH, Vogels DS. Study of orthodontic diagnosis and treatment procedures. J ClinOrthod1991; 25:145-56.

2. Salonen L, Mohlin B, Götzlinger B, Helldén L. Need and demand for orthodontic treatment in an adult Swedish population. Eur J Orthod1992; 14:359-68.

3. Nattrass C, Sandy JR. Adult orthodontics - a review. Br J Orthod1995; 22:331-7. 


\section{Hassan BA}

4. Roeters JJ. Extended indications for directly bonded composite restorations: a clinician's view. J Adhes Dent 2001; 3:81-7. 5. Lilja-Karlander E, Kurol J, Josefsson E. Attitudes and satisfaction with dental appearance in young adults with and without malocclusion. Swed Dent J 2003; 27:143-50 6. Mizrahi B. A technique for simple and aesthetic treatment of anterior tooth wear. Dent Update 2004; 31:109-114.

7. Crumpler DC, Bayne SC, Sockwell S, Brunson D, Roberson TM. Bonding to resurfaced posterior composites. Dent Mater 1989; 5:417-24.

8.Livaditis GJ, Thompson VP. Etched castings: an improved retentive mechanism for resin-bonded retainers. J Prosthet Dent 1982; 47:52-8.

9. Newman SM, Dressler KB, Grenadier MR. Direct bonding of orthodontic brackets to esthetic restorative materials using a silane. Am J Orthod Dentofacial Orthop1984; 86:503-6.

10.Livaditis GJ. A chemical etching system for creating micromechanical retention in resin-bonded retainers. J Prosthet Dent 1986; 56:181-8.

11. Gillis I, Redlich M. The effect of different porcelain conditioning techniques on shear bond strength of stainless steel brackets. Am J Orthod Dentofacial Orthop1998; 114:387-92.

12. Zachrisson BU. Orthodontic bonding to artificial tooth surfaces: clinical versus laboratory findings. Am J Orthod Dentofacial Orthop2000; 117:592-94.

13. Heyman H, Swift JR, Ritter A. Sturdevant's Art and Science of Operative dentistry. St. Louis: Elsevier, 2013.

14. Farzaegan F, Tanbakuchi B. Are Bonding Agents being Effective on the Shear Bond Strength of Orthodontic Brackets Bonded to the Composite? JDMT 2014; 3(2):61-5.

15. Özcan M, Vallittu PK, Peltomäki T. Bonding polycarbonate brackets to ceramic: effects of substrate treatment on bond strength. Am J Orthod Dentofacial Orthop 2004; 126:220-7.

16. Phiton MM, Oliveira MV, Ruellas AC. Shear bond strength of orthodontic brackets to enamel under different surface treatment conditions. J Appl Oral Sci 2007;15:127-30. 17. Valleta R, Prisco D, Ambrosio L, Martina R. Evaluation of the debonding strength of orthodontic brackets using three different bonding systems. Eur J Orthod 2007;29:571-7.

18. T. Hayakawa, K. Horie, M. Aida, H. Kanaya, T. Koba-yashi and Y. Murata, "The Influence of Surface Conditions and Silane Agents on the Bond of Resin to Dental Porcelain," Dental Materials, Vol. 8, No. 4, 1992, pp. 238-240.

19. Eslamian L, Borzabadi-Farahani A, Mousavi N, Ghasemi A. The effects of various surface treatments on the shear bond strengths of stainless steel brackets to 
artificially-aged composite restorations. AustOrthod J 2011; 27:28-32.

20. Chay SH, Wong SL, Mohamed N, Chia A, Yap AU. Effects of surface treatment and aging on the bond strength of orthodontic brackets to provisional materials. Am J Orthod Dentofacial Orthop 2007; 132:57781.

21. Atsü SS, Gelgör IE, Sahin V. Effects of silica coating and silane surface conditioning on the bond strength of metal and ceramic brackets to enamel. Angle Orthod 2006; 76:857-62.

22. Eustaquio R, Garner LD, Moore BK. Comparative tensile strengths of brackets bonded to porcelain with orthodontic adhesive and porcelain repair systems. Am $\mathrm{J}$ Orthod Dentofacial Orthop 1988; 94:421-5.

23. Zachrisson BU. Orthodontic bonding to artificial tooth surfaces: clinical versus laboratory findings. Am J Orthod Dentofacial Orthop 2000; 117:592-4.

24. Zachrisson $\mathrm{Y}$, Zachrisson BU, Büyükyilmaz T. Surface preparation for orthodontic bonding to porcelain. Am J Orthod Dentofacial Orthop 1996; 109:42030.

25. Smith GA, Mcinnes-Ledoux P, Ledoux WR. Orthodontic bonding to porcelainBond strength and refinishing. Am J Orthod Dentofacial Orthop 1988; 94:245-52.

26. Spohr AM, Sobrinho LC, Consani S, Sinhoreti MA, Knowles JC. Influence of surface conditions and silane agent on the bond of resin to IPS Empress 2 ceramic. Int J Prosthodont2003; 16:277-282.

27. Chung $\mathrm{CH}$, Cuozzo PT, Mante FK. Shear bond strength of a resin-reinforced glass ionomer cement: an in vitro comparative study. Am J Orthod Dentofacial Orthop1999; 115:52-54.

28. Jabber $\mathrm{Z}$ A. Evaluation the Effect of Different Surface Treatment on Shear Bond Strength between Composite Increments (An in vitro study). IJSRP 2017; 7(1): 67685.

29. Barbosa VLT, Almeida MA, Chevitarese O. Direct bonding to porcelain. Am J Orthod Dentofacial Orthop 1995; 107:159-64.

30. Schmage P, Nergiz I, Herrmann W. Influence of various surface-conditioning methods on the bond strength of metal brackets to ceramic surfaces. Am J Orthod Dentofacial Orthop 2003; 123:540-6.

31. Newman SM, Dressler KB, Grenadier MR. Direct bonding of orthodontic brackets to esthetic restorative materials using a silane. Am J Orthod Dentofacial Orthop 1984; 86:503-6.

32. Eslamian L, Ghassemi A, Amini F, Jafari A, Afrand M. Should silane coupling agents be used when bonding brackets to composite restorations? An in vitro study. Eur J Orthod2009; 31:266-70.

33. Kocadereli I, Canay S, Akça K. Tensile bond strength of ceramic orthodontic brackets bonded to porcelain surfaces. Am J 


\section{Hassan BA}

Orthod Dentofacial Orthop 2001; 119:61720.

34. Nebbe B, Stein E. Orthodontic brackets bonded to glazed and deglazed porcelain surfaces. Am J Orthod Dentofacial Orthop 1996; 109:431-6.

35. Zachrisson BU, Buyukyilmaz T. Recent advances in bonding to gold, amalgam and porcelain. J Clinic Orthod 1993; 27:661-74.

36. Huang TH, Kao CT. The shear bond strength of composite brackets on porcelain teeth. Eur J Orthod 2001; 23:433-9.

37. Bourke BM, Orth FDSM, Rock WP. Factors affecting the shear bond strength of orthodontic brackets to porcelain. $\mathrm{Br} \mathrm{J}$ Orthod 1999; 26:285-90. 